\title{
SOBREVIVIR A LA INVASIÓN DE LOS PAÍSES DEL NUEVO MUNDO: LA ESTRATEGIA EXPORTADORA GANADORA DE LA INDUSTRIA VITIVINÍCOLA ESPAÑOLA
}

\author{
$\underline{\text { Juan Ramón Ferrer }}^{\mathbf{a}^{(*)}}$, Raúl Serrano ${ }^{\mathrm{b}}$, Silvia Abella ${ }^{\mathrm{b}}$, Vicente Pinilla ${ }^{\mathrm{b}}$ y María Teresa Maza $^{\mathrm{b}}$ \\ ${ }^{a}$ Universidad Politécnica de Madrid (Madrid, juanramon.ferrer@upm.es). \\ b Universidad de Zaragoza (raser@unizar.es, sabella@unizar.es, vpinilla@unizar.es, \\ mazama@unizar.es)
}

\begin{abstract}
Resumen
El artículo explora la influencia de la estrategia de las empresas en el desempeño exportador en la industria del vino en España. La literatura previa se ha centrado en analizar los resultados exportadores en el mercado del vino con base, sobre todo, en aspectos exógenos a las empresas, prestando menor atención a esta caja negra base del conjunto de decisiones empresariales. Los resultados de este estudio muestran un modelo estratégico más complejo que el dicotómico de la teoría de Porter. El uso conjunto de estrategias, que buscan la eficiencia (a través de la reducción de costos), al tiempo que incrementan el valor para el cliente (a través de la innovación y la mejora de los procesos de comunicación y distribución), logran un incremento en la internacionalización de la empresa. Utilizando la metodología Heckman, analizamos las variables que determinan la decisión de exportar y la intensidad exportadora, a partir de 339 empresas vitivinícolas españolas. El acercamiento a la estrategia de las empresas se realiza mediante el modelo de Robinson y Pearce. Los resultados revelan que, para expandirse en los mercados internacionales, las empresas pueden utilizar una combinación de diferentes opciones estratégicas (eficiencia, innovación y marketing), sin considerar estas opciones como alternativas antagónicas.
\end{abstract}

Palabras clave: estrategias, competitividad, mercado de exportación, sector del vino

\section{Introducción}

El mercado mundial del vino ha cambiado profundamente desde mediados de la década de los ochenta del siglo XX. Por un lado, ha tenido lugar una auténtica globalización de su consumo, lo que ha supuesto un claro incremento de la demanda en lugares donde ésta era hasta entonces muy reducida y un fortísimo crecimiento de su comercio. Por otro lado, la irrupción de los conocidos como países del Nuevo Mundo (USA, Chile, Australia, Argentina y Sudáfrica) como exportadores importantes ha supuesto un notable desafío para los tradicionales exportadores del suroeste europeo (Francia, Italia y España), que han visto reducir significativamente su participación en el mercado mundial. En este contexto, llama la atención el dinamismo de las exportaciones españolas de vino que, frente a la amenaza representada por los nuevos países presentes en el mercado, ha conseguido incrementar significativamente su volumen exportado y su peso en el mercado internacional.

Como han puesto de relieve numerosos trabajos, los resultados exportadores dependen de aspectos macroeconómicos y de las políticas comerciales (Anderson and Wittwer 2018). Pero el resultado de la exportación también va a depender de la acción de las empresas, de sus decisiones, de su capacidad de adaptación. La capacidad de exportación se va a ver influida por las decisiones estratégicas de las empresas. Una caja negra poco explorada y que en las últimas décadas ha sido considerada como clave en la determinación de una exitosa estrategia de comercio internacional (Bargain , 2020).

Consideramos que hasta ahora se ha desarrollado una importante literatura centrada en analizar los resultados exportadores en el mercado del vino con base, sobre todo, en aspectos exógenos a las empresas, prestándose mucha menor atención al impacto de sus estrategias (Anderson and Witwer, 2020). En este contexto es necesario explicar el fuerte crecimiento de las exportaciones españolas de vino, y particularmente el de vino embotellado procedente de las denominaciones de origen protegidas, en los últimos veinte años (Fernandez y Pinilla, 2018). Indudablemente se puede adoptar una perspectiva macroeconómica con la utilización de modelos de gravedad para tratar de averiguar cuáles son los factores determinantes del crecimiento exportador. Numerosos trabajos han adoptado esta metodología para diferentes países y periodos, proporcionando resultados de indudable interés. El objetivo de este trabajo es conocer: ¿Qué estrategias favorecen el hecho de exportar? ¿Qué estrategias favorecen un mayor volumen de exportación?

\section{Objetivos}

El objetivo de este artículo es tratar de llenar el vacío existente analizando las estrategias empresariales genéricas y su impacto en el incremento de sus exportaciones. Se utiliza muestra de 339 empresas vinícolas 
españolas. La aproximación a las estrategias de las empresas se realiza a través del modelo de Robinson y Pearce (1988) y la metodología utilizada el modelo de Heckman de dos etapas.

\section{Metodología}

El universo de empresas utilizadas en el estudio está formado por bodegas españolas derivadas de la combinación de dos bases de datos diferentes. El primero contiene información sobre las DOP e IGP, recopilada por el Ministerio de Agricultura, Pesca y Alimentación (MAGRAMA); el segundo es el Sistema de Análisis de Balances Ibéricos (SABI), del que hemos tomado las empresas pertenecientes al apartado 11.02 de la Clasificación de Actividades Económicas (CNAE). El tamaño de la población es de 2.413 bodegas. Con el fin de obtener la información necesaria para el análisis, se envió un cuestionario a los gerentes de estas firmas solicitando información sobre la situación de las firmas en diciembre de 2015. El proceso se desarrolló entre febrero y mayo de 2016. Hubo un total de 339 respuestas, de las que un 14,7\% no exportan.

Para estudiar la relación entre las variables, utilizamos un modelo dinámico de Heckman-Probit que corrige los problemas relacionados con el sesgo de selección. El primer paso analiza las estrategias que influyen en la decisión de las empresas de exportar. En el segundo paso, analizamos los factores que influyen en la intensidad exportadora de estas empresas. De esta forma intentamos verificar el efecto de diferentes tipos de estrategias. Las variables independientes son, en la primera etapa, si la empresa exporta o no exporta (variable dummy, ecuación 1). Y en la segunda etapa la intensidad de la exportación (ecuación 2)

$\mathrm{D}^{\exp _{\mathrm{i}, \mathrm{t}}=}\left\{\begin{array}{l}1 \text { (export) } \Longrightarrow \mathrm{P}\left(\mathrm{D}_{\mathrm{i}, \mathrm{t}}=1\right)=\mathrm{f}(\text { Estrategias }+ \text { variables de control }) \\ 0 \text { (no export }), \text { en otro caso }\end{array}\right.$

Variables independientes, la estrategia se configura a través de 22 cuestiones sobre comportamientos empresariales, a las que contestan los gerentes de las empresas, con ello se delimitan cuatro estrategias genéricas, eficiencia, servicio, innovación y marketing (Robinson y Pearce, 1988). (Ver cuadro 1)

Cuadro 1. Cuestiones de comportamiento empresarial y estrategias genéricas (Robinson y Pearce, 1988)

\begin{tabular}{|c|c|c|}
\hline Estrategia genéricas & $\begin{array}{l}\text { Cuestiones de comportamiento } \\
\text { (Robinson y Pearce,1988) }\end{array}$ & Comentarios e interpretación \\
\hline \multirow[t]{6}{*}{$\begin{array}{l}\text { Eficiencia } \\
\text { (bajo precio) }\end{array}$} & $\begin{array}{l}\text { Esfuerzos específicos para asegurar un } \\
\text { grupo de personal experimentado y } \\
\text { altamente capacitado }\end{array}$ & \multirow[t]{6}{*}{$\begin{array}{l}\text { Esfuerzo para asegurar operaciones } \\
\text { eficientes y rentables. }\end{array}$} \\
\hline & $\begin{array}{l}\text { Procedimientos de estricta calidad y } \\
\text { control }\end{array}$ & \\
\hline & Énfasis en mejorar el costo por unidad & \\
\hline & $\begin{array}{l}\text { Innovación en los procesos de } \\
\text { fabricación }\end{array}$ & \\
\hline & $\begin{array}{l}\text { Innovación en técnicas y métodos de } \\
\text { marketing }\end{array}$ & \\
\hline & Precio por debajo de la competencia $*$ & \\
\hline \multirow[t]{5}{*}{$\begin{array}{l}\text { Servicio (alto } \\
\text { precio) }\end{array}$} & $\begin{array}{l}\text { No se tiene preocupación por tener } \\
\text { precios inferiores a la competencia (se } \\
\text { usa en negativo) }\end{array}$ & \multirow{5}{*}{$\begin{array}{l}\text { Proporcionar un amplio servicio a los } \\
\text { clientes, mercados de precios altos, } \\
\text { desarrollo de una reputación en la } \\
\text { industria }\end{array}$} \\
\hline & Servicio al cliente & \\
\hline & $\begin{array}{l}\text { Preocupación por construir una imagen } \\
\text { de reputación de marca } \\
\text { within }\end{array}$ & \\
\hline & $\begin{array}{l}\text { Productos en el segmento de alto } \\
\text { precio }\end{array}$ & \\
\hline & $\begin{array}{l}\text { Se evitan los segmentos de bajo } \\
\text { precio }\end{array}$ & \\
\hline \multirow{2}{*}{$\begin{array}{l}\text { I+D (Innovación en el } \\
\text { desarrollo de } \\
\text { productos) }\end{array}$} & Desarrollo de nuevos productos & \multirow{2}{*}{$\begin{array}{l}\text { Productos especializados y nuevos } \\
\text { desarrollos o refinamientos basados en } \\
\text { parte en procesos de I+D. }\end{array}$} \\
\hline & $\begin{array}{l}\text { Desarrollo y evolución de los productos } \\
\text { existentes }\end{array}$ & \\
\hline
\end{tabular}




\begin{tabular}{|c|c|c|}
\hline & $\begin{array}{l}\text { Énfasis en el manufacturado de } \\
\text { productos especiales }\end{array}$ & \\
\hline & $\begin{array}{l}\text { Incremento en la inversión en procesos y } \\
\text { orientación al I+D }\end{array}$ & \\
\hline \multirow{4}{*}{$\begin{array}{l}\text { Marketing } \\
\text { (Marca/ influencia en } \\
\text { el canal) }\end{array}$} & Identificación de marca & \multirow{4}{*}{$\begin{array}{l}\text { Reconocimiento de marca y una fuerte } \\
\text { influencia sobre los canales a través de } \\
\text { esfuerzos como el desarrollo de productos } \\
\text { y nuevas técnicas de marketing. }\end{array}$} \\
\hline & $\begin{array}{l}\text { Fuerte influencia sobre los canales de } \\
\text { distribución }\end{array}$ & \\
\hline & Desarrollo de nuevos productos & \\
\hline & $\begin{array}{l}\text { Innovación en técnicas y métodos de } \\
\text { marketing }\end{array}$ & \\
\hline
\end{tabular}

Fuente: Robinson y Pearce (1988). (*) Incorporado por los autores

Como variables de control se incluyen, el tamaño, la edad, la disposición de recursos humanos, la participación en proyectos de I+D, y una variable dummy sobre su pertenencia a un grupo empresarial. Que quedan reflejadas en la ecuación (2). En todos los modelos que se van a desarrollar se mantiene la estrategia de servicio por haber sido relacionada profusamente con la exportación (Crozet et al, 2011).

Export intensity $_{i}=\beta_{1}$ Strategies $_{i}+\beta_{2}$ Size $_{i}+\beta_{3}$ Age $e_{i}+\beta_{4} R \& D_{i}+\beta_{5}$ HumanResourses $_{i}+\beta_{6}$ Business

$$
\text { Group }_{i}+U_{i}
$$

\section{Resultados}

Con el objeto de disponer de resultados que muestren, no tan solo si las estrategias genéricas favorecen la exportación sino también si su combinación es un elemento explicativo de un mayor volumen de exportación, se han desarrollado diferentes modelos. Las combinaciones posibles son muchas y el desarrollo de todas las opciones, excede el ámbito de este trabajo. Se plantean diferentes modelos que investigan el efecto multiplicativo de las estrategias y que se presentan según las distintas fases que la investigación asume. Fase 1: ¿Qué hace que las empresas exporten? Para ello se analizan estrategia de servicio con el resto de las estrategias genéricas (modelos 1, 2 y 3; con las dos etapas del Heckman), columnas 1 a 6 del cuadro 2. Los resultados de la fase 1 demuestran que todas las estrategias favorecen que las empresas exporten (columnas 1 a 3 del cuadro2), pero ninguna de manera independiente explica un mayor volumen de exportación (columnas 4 a 6 del cuadro 2). Fase 2, ¿Es posible que la combinación de estrategias provoque un mayor volumen de exportación? Para ello, se analizan manteniendo la estrategia de diferenciación como variable independiente, tres modelos con dos estrategias multiplicativas, modelos 4 (eficiencia x marketing), 5 (eficiencia x innovación) y 6 (marketing x innovación). Y se complementa con un modelo 7, en que se analiza el efecto multiplicativo de las tres estrategias, eficiencia, marketing e innovación. Se observa, como la combinación de estrategias mejora el desempeño exportador de las empresas. Cabe destacar el efecto positivo en el volumen de exportación de una combinación de estrategias de innovación y eficiencia (columna 8) y la combinación de estrategias de eficiencia, innovación y marketing (columna 10).

Cuadro 2. Desarrollo modelo de Heckman

\begin{tabular}{|c|c|c|c|c|c|c|c|c|c|c|}
\hline Variable & $\begin{array}{c}(1) \\
\text { Etapa } 1 . \\
\text { Modelo } 1\end{array}$ & $\begin{array}{c}(2) \\
\text { Etapa } 1 \\
\text { Modelo 2 }\end{array}$ & $\begin{array}{c}(3) \\
\text { Etapa } 1 \\
\text { Modelo 3 }\end{array}$ & $\begin{array}{c}(4) \\
\text { Etapa } 2 \\
\text { Modelo } 1\end{array}$ & $\begin{array}{c}(5) \\
\text { Etapa 2 } \\
\text { Modelo 2 }\end{array}$ & $\begin{array}{c}(6) \\
\text { Etapa 2 } \\
\text { Modelo 3 }\end{array}$ & $\begin{array}{c}(7) \\
\text { Etapa 2 } \\
\text { Modelo } 4\end{array}$ & $\begin{array}{c}(8) \\
\text { Etapa 2 } \\
\text { Modelo 5 }\end{array}$ & $\begin{array}{c}(9) \\
\text { Etapa } 2 \\
\text { Modelo 6 }\end{array}$ & $\begin{array}{c}(10) \\
\text { Etapa } 2 \\
\text { Modelo } 7\end{array}$ \\
\hline Servicio & $\begin{array}{c}0.135 \\
* * *\end{array}$ & $0.149 * * *$ & $\begin{array}{c}0.148 \\
* * *\end{array}$ & 0.016 & -0.152 & -0.215 & -0.115 & -0.239 & -0.076 & -0.054 \\
\hline Eficiencia & $\begin{array}{c}0.094 \\
* * *\end{array}$ & & & 0.017 & & & & & & \\
\hline Marketing & & $0.048^{* * * *}$ & & & 0.193 & & & & & \\
\hline Innovación & & & 0.040 ** & & & 0.263 & & & & \\
\hline $\begin{array}{l}\text { Eficiencia * } \\
\text { Marketing }\end{array}$ & & & & & & & 0.041 & & & \\
\hline $\begin{array}{l}\text { Eficiencia * } \\
\text { Innovación }\end{array}$ & & & & & & & & $0.060 * * *$ & & \\
\hline $\begin{array}{l}\text { Marketing* } \\
\text { Innovación }\end{array}$ & & & & & & & & & $0.054^{*}$ & \\
\hline
\end{tabular}




\begin{tabular}{|c|c|c|c|c|c|c|c|c|c|c|}
\hline $\begin{array}{l}\text { Eficiencia * } \\
\text { Marketing * } \\
\text { Innovación }\end{array}$ & & & & & & & & & & $0.016^{* *}$ \\
\hline Tamaño & $0.017 * *$ & $0.030 * * *$ & $0.032 * * *$ & $0.259 * *$ & $0.248 * *$ & $0.263 * *$ & $0.296^{* *}$ & $0.410^{* * * *}$ & $0.319 * *$ & $0.342 * * *$ \\
\hline Antiguedad & $0.001 * *$ & $0.030 * *$ & $0.001 * *$ & -0.003 & -0.000 & 0.000 & 0.000 & 0.000 & -0.000 & 0.001 \\
\hline$I+D$ & -0.005 & -0.003 & -0.003 & 0.076 & 0.027 & 0.002 & -0.001 & -0.027 & 0.011 & -0.012 \\
\hline $\begin{array}{l}\text { Recursos } \\
\text { humanoss } \\
\end{array}$ & $0.035 * *$ & $0.049 * * *$ & $0.056 * * *$ & 0.109 & 0.150 & 0.165 & 0.170 & $0.226^{* *}$ & 0.062 & 0.049 \\
\hline $\begin{array}{l}\text { Grupo } \\
\text { empresarial }\end{array}$ & -0.004 & -0.028 & -0.027 & -0.117 & -0.050 & -0.140 & -0.313 & -0.399 & -0.563 & -0.731 \\
\hline Inversa Mills & $\cdots$ & $\cdots$ & $\cdots$ & $\cdots$ & $\cdots$ & $\cdots$ & $\cdots$ & $\cdots$ & $\cdots$ & $\cdots$ \\
\hline Constante & $\cdots$ & $\cdots$ & $\cdots$ & $\cdots$ & $\cdots$ & $\cdots$ & $\cdots$ & $\cdots$ & $\cdots$ & $\cdots$ \\
\hline $\begin{array}{l}\text { Observaciones } \\
\text { Prob > chi2 }\end{array}$ & $\begin{array}{c}267 \\
0.000\end{array}$ & $\begin{array}{c}267 \\
0.000\end{array}$ & $\begin{array}{c}269 \\
0.000\end{array}$ & $\begin{array}{c}229 \\
0.000\end{array}$ & $\begin{array}{c}228 \\
0.000\end{array}$ & $\begin{array}{c}230 \\
0.000\end{array}$ & $\begin{array}{c}232 \\
0.000\end{array}$ & $\begin{array}{c}232 \\
0.000\end{array}$ & $\begin{array}{c}232 \\
0.000\end{array}$ & $\begin{array}{c}232 \\
0.000\end{array}$ \\
\hline
\end{tabular}

\section{Conclusiones}

Los resultados validan el éxito de la combinación de estrategias para incrementar la intensidad exportadora de la industria vitivinícola, y especialmente, la combinación innovación y eficiencia.

\section{Bibliografía}

Anderson, K. and Wittwer, G. (2020). "A model of global beverage markets”. Journal of Wine Economics, 15(3), 330-354.

Anderson, K., and Wittwer, G. (2018). "Cumulative effects of Brexit and other UK and EU-27 bilateral free-trader agreements on the world's wine markets". The World Economy, 41 2883-2894.

Bargain, O. (2020). "French wine exports to China: evidence from intra-French regional diversification and competition". Journal of Wine Economics, 15(2), 134-162.

Crozet, M., Head, K., \& Mayer, T. (2011). "Quality sorting and trade: Firm-level evidence for French wine". The Review of Economic Studies, 79(2), 609-644.

Fernández, E., and Pinilla, V. (2018). “Spain”. En K. Anderson and V. Pinilla (eds.), Wine Globalization: A New Comparative History, 208-238. New York: Cambridge University Press.

Robinson, R. B., and Pearce, J. A. (1988). "Planned patterns of strategic behavior and their relationship to business-unit performance”. Strategic Management Journal, 9(1), 43-60. 